\title{
LIMIT POINTS IN THE RANGE OF THE COMMUTING PROBABILITY FUNCTION ON FINITE GROUPS
}

\author{
PETER HEGARTY
}

\begin{abstract}
If $G$ is a finite group, then $\operatorname{Pr}(G)$ denotes the fraction of ordered pairs of elements of $G$ which commute. We show that if $l \in\left(\frac{2}{9}, 1\right]$ is a limit point of the function $\operatorname{Pr}$ on finite groups, then $l \in \mathbb{Q}$ and there exists an $\epsilon=\epsilon_{l}>0$ such that $\operatorname{Pr}(G) \notin\left(l-\epsilon_{l}, l\right)$ for any finite group $G$. These results lend support to some old conjectures of Keith Joseph.
\end{abstract}

\section{INTRODUCTION}

Let $\mathcal{G}$ denote the family of all finite groups and define the function Pr: $\mathcal{G} \rightarrow \mathbb{Q} \cap(0,1]$ as follows]: for $G \in \mathcal{G}$,

$$
\operatorname{Pr}(G):=\frac{\#\{(x, y) \in G \times G: x y=y x\}}{|G|^{2}} .
$$

We call $\operatorname{Pr}(G)$ the commuting probabilit) 2 $^{2}$ for $G$, in other words it is the probability that a uniformly random ordered pair of elements of $G$ commute. It is easy to see that $\operatorname{Pr}(G)=k(G) /|G|$, where $k(G)$ denotes the number of conjugacy classes in $G$. There is quite a lot of literature on the properties of the function Pr. Much of this dates from a decade-or-so long period from the late 1960s to the late 1970s. In 1968, Erdôs and Turán $[\mathrm{ET}]$ and, independently, Newman $[\mathrm{New}]$, proved that, for any finite group $G$,

$$
\operatorname{Pr}(G) \geq \frac{\log _{2} \log _{2}|G|}{|G|} .
$$

This is a quantitative version of the classical fact that there are only finitely many finite groups with a given number of conjugacy classes. There have been various improvements on (1.2) since, as well as improved lower bounds for particular classes of groups: see $[\mathrm{Ke}]$ and the references therein. It is easy to prove, as suggested by (1.2), that $\operatorname{Pr}(G)$ can be arbitrarily close to zero. On the other hand, it is obvious that $\operatorname{Pr}(G)=1$ if and only if $G$ is abelian. Gustafson [G] seems to have been the first to record, in 1973, the fact that, if $G$ is non-abelian, then $\operatorname{Pr}(G) \leq 5 / 8$, with equality being achieved if and only if $G / Z(G) \cong C_{2} \times C_{2}$. The intervening years have witnessed a minor flood of papers whose common theme is to show that, if $\operatorname{Pr}(G)$ is sufficiently large, then $G$ is

\footnotetext{
Date: October 21, 2018.

2000 Mathematics Subject Classification. 20D99, 20E34, 20E45, $20 \mathrm{P} 99$.

Key words and phrases. Commuting probability, limit points.

${ }^{1}$ Various alternative notations for this function appear in the literature, for example: $\operatorname{cp}(G), \operatorname{mc}(G)$, $d(G)$.

${ }^{2}$ Some authors, including for example Lescot, who has written a number of papers about this function (see $[\mathrm{L}]$ ), calls it the commutativity degree rather than the commuting probability.
} 
"close to abelian" in some well-defined structural sense. There are basically two types of results here:

(i) Rusin $[\mathrm{R}]$ completely classifies all finite groups for which $\operatorname{Pr}(G)>11 / 32$. A recent work of Das and Nath [DN] does the same 3 for all odd order groups satisfying $\operatorname{Pr}(G) \geq 11 / 75$. The point here is that one can completely determine all finite groups $G$ for which $\operatorname{Pr}(G)$ lies above a certain threshold.

(ii) There are also results of a more general character, whose purpose is to determine some general "abelian-like feature" of a group $G$ for which $\operatorname{Pr}(G)$ is bounded away from zero. A fairly recent work of Guralnick and Robinson [GR] contains a number of impressive such results. In particular, they prove ([GR], Theorem 10) that

$$
\operatorname{Pr}(G) \leq(G: F)^{-1 / 2}
$$

where $F$ is the Fitting subgroup of $G$. Hence, if $\operatorname{Pr}(G)$ is bounded away from zero, then $G$ contains a normal, nilpotent subgroup of bounded index. A similar, but even more striking result was shown much earlier by Peter Neumann:

Theorem 1.1 (P.M. Neumann [Neu]). For each $\epsilon>0$, there exist positive integers $n_{1}(\epsilon), n_{2}(\epsilon)$ such that the following holds: If $G$ is a finite group satisfying $\operatorname{Pr}(G)>\epsilon$, then $G$ possesses normal subgroups $H, K$ such that

(i) $K \subseteq H$,

(ii) $|K| \leq n_{1}(\epsilon)$,

(iii) $(G: H) \leq n_{2}(\epsilon)$,

(iv) $H / K$ is abelian.

In this paper we are basically interested in the following question:

What is the range of the function Pr inside $\mathbb{Q} \cap(0,1]$ ?

Gustafson's observation makes this question very natural, as it implies that the range is not the whole of $\mathbb{Q} \cap(0,1]$. This is in stunning contrast to the situation for semigroups: see $[\overline{\mathrm{PS}}]$. In the case of groups, further insight into the range of Pr can be gleaned, if indirectly, from the various papers cited above. As far as we know, however, only one person ever seems to have seriously considered general structural properties of $\operatorname{Pr}(\mathcal{G})$ as a set. Keith Joseph wrote his Ph.D. thesis [J1] in 1969 on the commuting probability, but it was never published. Eight years later, however, in a short note in the Monthly [J2], he posed three very interesting conjectures about the range of Pr, which we reproduce below 4 . Note that, by a limit point of a set $S \subseteq \mathbb{R}$, we mean an element $l \in \mathbb{R}$ such that there is a sequence $\left(s_{n}\right)$ of elements of $S$ tending to $l$. In particular, every element of $S$ itself is a limit point of $S$.

Joseph's first conjecture. Every limit point of $\operatorname{Pr}(\mathcal{G})$ is rational. Joseph's second conjecture. If $l$ is a limit point of $\operatorname{Pr}(\mathcal{G})$, then there exists $\epsilon=\epsilon_{l}>0$ such that $\operatorname{Pr}(\mathcal{G}) \cap\left(l-\epsilon_{l}, l\right)=\phi$.

\footnotetext{
${ }^{3}$ There were some errors in Rusin's paper, which were pointed out and corrected by Das-Nath. See also the proof of Lemma 2.1 below.

${ }^{4}$ The second and third conjectures were stated slightly differently by Joseph.
} 
Joseph's third conjecture. $\operatorname{Pr}(\mathcal{G}) \cup\{0\}$ is a closed subset of $\mathbb{R}$.

Unfortunately, and despite the large amount of literature in existence today on the commuting probability, Joseph's conjectures seem to have suffered the same fate as the rest of his work and been thoroughly neglected 5 . There seems to have been essentially no progress on any of them, and we found only a handful of references to his work. For example, Rusin $[\underline{R}]$ refers to Joseph's questions at the end of his paper. He notes that, since his methods rely heavily on the elementary estimate

$$
\operatorname{Pr}(G) \leq \frac{1}{4}+\frac{3}{4} \frac{1}{\left|G^{\prime}\right|}
$$

they cannot, even in principle, yield any insight into the range of Pr inside $(0,1 / 4]$. Rusin wonders explicitly whether the range is dense inside $(1 / 5,1 / 4)$. In a recent Master's thesis, Castelaz $\sqrt{6}([\vec{C}]$, end of Chapter 5$)$ essentially repeats Joseph's questions, but does not provide any new insights. Only the following facts seem to be known, and all but the last appear to have been already known to Joseph. For proofs of (i)-(iv), see [C]:

(i) zero is a limit point, not in the range of Pr.

(ii) for each prime $p, 1 / p$ is a limit point of $\operatorname{Pr}(\mathcal{G})$ and there exists a group $G$ with $\operatorname{Pr}(G)=1 / p$.

(iii) since the function $\operatorname{Pr}$ is multiplicative, i.e.:

$$
\operatorname{Pr}\left(G_{1} \times G_{2}\right)=\operatorname{Pr}\left(G_{1}\right) \times \operatorname{Pr}\left(G_{2}\right),
$$

it follows that the same is true as in (ii) for every number $a / n \in(0,1]$, such that $n \in \mathbb{N}$ and $a \in \operatorname{Pr}(\mathcal{G})$.

(iv) Joseph's second conjecture is known to hold for $q=1 / 2$ (with $\epsilon_{1 / 2}=1 / 16$ ).

(v) The following is a corollary of a result of Gavioli et al:

Theorem 1.2 (Corollary to Theorem 3 in [GMMPS]). Let $p \geq 7$ be a prime and let $G$ be a p-group. Then

$$
\operatorname{Pr}(G) \notin\left[\frac{5}{p^{4}}, \frac{1}{p^{3}}\right] .
$$

In this paper we shall provide some new evidence for Joseph's first two conjectures by proving the following result:

Main Theorem. If $l \in\left(\frac{2}{9}, 1\right]$ is a limit point of the set $\operatorname{Pr}(\mathcal{G})$, then

(i) $l \in \mathbb{Q}$,

(ii) there exists an $\epsilon=\epsilon_{l}>0$ such that $\operatorname{Pr}(\mathcal{G}) \cap\left(l-\epsilon_{l}, l\right)=\phi$.

Note that, as stated in (iv) above, the second assertion in our theorem is already known for $l=1 / 2$. As well as giving a bit of new evidence in support of Joseph's long-dormant program, our result provides the first insight into the range of Pr below 1/4, for arbitrary

\footnotetext{
${ }^{5}$ As already mentioned, his Ph.D. thesis was never published. Neither is he listed as an author on MathSciNet. His Monthly article appeared as a "research problem", not as a regular paper.

${ }^{6}$ who has since married and appears on MathSciNet as Anna Keaton, see:

https://mthsc.clemson.edu/directory/viewperson.py?personid=198
} 
groups (Theorem 1.2 gives some insight for $p$-groups and the results in [DN] for odd order groups), as sought by Rusin. After some preliminaries in Section 2, we will prove our theorem in Section 3. In Section 4 we will discuss the prospects for extending our methods in the hope of answering Joseph's questions definitively.

\section{Preliminaries}

Throughout the rest of this paper, all groups are finite unless explicitly stated otherwise. Recall that groups $G_{1}$ and $G_{2}$ are said to be isoclinic if there are isomorphisms

$$
\phi: G_{1} / Z_{1} \rightarrow G_{2} / Z_{2}, \quad \psi: G_{1}^{\prime} \rightarrow G_{2}^{\prime} \quad\left(Z_{1}=Z\left(G_{1}\right), Z_{2}=Z\left(G_{2}\right)\right),
$$

such that, for all $x, y \in G_{1}$,

$$
\psi\left(\left[Z_{1} x, Z_{1} y\right]\right)=\left[\phi\left(Z_{1} x\right), \phi\left(Z_{1} y\right)\right] .
$$

Isoclinism is an equivalence relation on the set of all finite groups and each equivalence class contains a so-called stem group, i.e.: a group $G$ such that $Z(G) \subseteq G^{\prime}$.

First, we collect a number of facts about the function Pr:

Lemma 2.1. (i) If $G$ is a non-abelian group then $\operatorname{Pr}(G) \leq 5 / 8$. Moreover, if $\operatorname{Pr}(G)>$ $11 / 32$ then one of the following must hold:

(a) $\left|G^{\prime}\right|=2, G / Z(G) \cong C_{2}^{2 s}$ for some $s \in \mathbb{N}$ and $\operatorname{Pr}(G)=\frac{1}{2} \cdot\left(1+2^{-2 s}\right)$.

(b) $G^{\prime} \cong C_{3}, G / Z(G) \cong S_{3}$ and $\operatorname{Pr}(G)=1 / 2$.

(c) $\operatorname{Pr}(G) \leq 7 / 16$ and $|G / Z(G)| \leq 16$.

(ii) Let $p$ be a prime and $G$ a p-group satisfying $G^{\prime} \subseteq Z(G)$. Then

$$
\operatorname{Pr}(G)=\frac{1}{\left|G^{\prime}\right|}\left(1+\sum_{K} \frac{(p-1)\left(G^{\prime}: K\right)}{p^{s(K)-1}}\right)
$$

where the sum is taken over all subgroups $K$ of $G^{\prime}$ for which $G^{\prime} / K$ is cyclic and the integer $n(K)$ is defined by

$$
p^{s(K)} \stackrel{\text { def }}{=} \frac{|G|}{|\{x \in G:[G, x] \subseteq K\}|} .
$$

In particular, if $G^{\prime} \cong C_{p}$ and $G / Z(G) \cong C_{p}^{2 s}$, then

$$
\operatorname{Pr}(G)=\frac{1}{p}\left(1+\frac{p-1}{p^{2 s}}\right)
$$

(iii) If $\left|G^{\prime}\right|=4$ and $\left|G^{\prime} \cap Z(G)\right|=2$ then

$$
\operatorname{Pr}(G)=\frac{1}{4}\left(1+\frac{1}{4}+\frac{1}{2^{2 s+1}}\right)
$$

where $2^{2 s}=\left[C_{G}\left(G^{\prime}\right): Z\left(C_{G}\left(G^{\prime}\right)\right)\right]$.

(iv) If $G^{\prime} \cong C_{6}$ and $G^{\prime} \cap Z(G) \cong C_{2}$ then $\operatorname{Pr}(G)=1 / 4+1 / 2^{\text {s }}$ for some $s \geq 3$.

(v) For any fixed group $K$, there are only finitely many groups $G$ satisfying $G^{\prime} \cong K$ and $Z(G)=\{1\}$. In particular, there are only finitely many possibilities for $\operatorname{Pr}(G)$ under these conditions. 
(vi) Let $G$ be a non-abelian group and let $d$ be the minimum degree of a non-linear irreducible representation of $G$. Then

$$
\frac{1}{\left|G^{\prime}\right|}<\operatorname{Pr}(G) \leq \frac{1}{d^{2}}+\left(1-\frac{1}{d^{2}}\right) \frac{1}{\left|G^{\prime}\right|} .
$$

(vii) If $H$ is a subgroup of $G$ then $\operatorname{Pr}(H) \geq \operatorname{Pr}(G)$.

(viii) If $N$ is a normal subgroup of $G$, then $\operatorname{Pr}(G) \leq \operatorname{Pr}(N) \cdot \operatorname{Pr}(G / N)$.

(ix) Let $N$ be a normal subgroup of $G$. Let $c$ be an integer such that every subgroup of $G / N$ contains at most c conjugacy classes. Let $k_{G}(N)$ denote the number of $G$-orbits of $N$, when $G$ acts on $N$ by conjugation. Then $k(G) \leq c \cdot k_{G}(N)$ and hence

$$
\operatorname{Pr}(G) \leq \frac{c}{|G / N|} \cdot \frac{k_{G}(N)}{|N|}
$$

(x) If the groups $G_{1}$ and $G_{2}$ are isoclinic, then $\operatorname{Pr}\left(G_{1}\right)=\operatorname{Pr}\left(G_{2}\right)$.

(xi) Let $n \in \mathbb{N}$ and let $\mathcal{Z}_{n}$ denote the collection of all finite groups $G$ for which

$(G: Z(G)) \leq n$. Then $\operatorname{Pr}\left(\mathcal{Z}_{n}\right)$ is a finite set.

Proof: Parts (i),(ii),(iv) and (v) can be found in [ $[\mathrm{R}]$. Part (iii) is proven in [DN], and they correct an erroneous form of (2.6) which appeared in $[\mathbb{R}]$. Parts (vi)-(ix) can be found in $[\mathrm{GR}]$ and part (x) in $[\mathrm{L}]$. We have not seen part (xi) written down anywhere (though similar statements appear in [NiS]), so we give the easy proof: Let $n$ be given and pick $G$ such that $(G: Z(G)) \leq n$. Then $\left|G^{\prime}\right|$ is bounded in terms of $n$, by Schur's classical result $[\mathbf{S}]$. Now, by part (vi), we may assume $G$ is a stem-group, in which case it follows that $|Z(G)|$ is also bounded. Thus $|G|$ is bounded, and hence there are only finitely many possible values of $\operatorname{Pr}(G)$.

Lemma 2.2. For elements $x, y, z, w$ in a group $G$ one has

$$
[x y, z w]=[x, w]^{y}[x, z]^{w y}[y, w][y, z]^{w} .
$$

PROOF: Simple exercise.

Lemma 2.3. Let $G$ be a finite subgroup of $P G L(2, \mathbb{C})$. Then $G$ is isomorphic to one of the following:

(i) a cyclic group $C_{n}$, for some $n \in \mathbb{N}$,

(ii) a dihedral group $D_{n}$ of order $2 n$, for some $n \geq 2$,

(iii) the tetrahedral group $A_{4}$,

(iv) the octahedral group $S_{4}$,

(v) the icosahedral group $A_{5}$.

Moroever, we have that

$$
\operatorname{Pr}\left(D_{n}\right)=\left\{\begin{array}{ll}
\frac{n+6}{4 n}, & \text { if } n \text { is even, } \\
\frac{n+3}{4 n}, & \text { if } n \text { is odd, }
\end{array} \quad \operatorname{Pr}\left(A_{4}\right)=\frac{1}{3}, \quad \operatorname{Pr}\left(S_{4}\right)=\frac{5}{24}, \quad \operatorname{Pr}\left(A_{5}\right)=\frac{1}{12} .\right.
$$

Proof: The classification of the finite subgroups of $\operatorname{PGL}(2, \mathbb{C})$ is classical; see, for example, [Kl]. The values of Pr for these groups can be easily verified. 
Before stating our last preliminary result, let us introduce some terminology which will simplify the statement of this and succeeding results:

Definition 2.1. A subset $S \subseteq \mathbb{R}$ will be called good if, for every limit point $l$ of $S$, the following hold:

(i) $l \in \mathbb{Q}$,

(ii) there exists $\epsilon=\epsilon_{l}>0$ such that $S \cap\left(l-\epsilon_{l}, l\right)=\phi$.

Observe that any subset of a union of finitely many good sets is also good.

Lemma 2.4. Let $n$ be a fixed positive integer and let

$$
\mathcal{S}_{n}:=\left\{\sum_{i=1}^{n} \frac{1}{x_{i}}: x_{i} \in \mathbb{N}\right\} .
$$

Then $\operatorname{Pr}\left(\mathcal{S}_{n}\right)$ is a good set.

ProOF: It is a classical fact that, for every fixed $n \in \mathbb{N}$ and $q \in \mathbb{Q}_{+}$, the equation

$$
\sum_{i=1}^{n} \frac{1}{x_{i}}=q
$$

has only finitely many positive integer solutions $\left(x_{1}, \ldots, x_{n}\right)$. If one examines the standard proof of this fact (which is basically just an induction on $n$ ), one easily sees that it in fact implies what is claimed in the lemma.

\section{Proof of MAin TheOrem}

The following lemma is the crucial ingredient in our proof, and we have not seen it written down before. The reader should observe the connection to Theorem 1.1, more about which will be said in the next section.

Lemma 3.1. Let $n \in \mathbb{N}$ and let $\mathcal{A}_{n}$ be the collection of all finite groups possessing a normal, abelian subgroup of index $n$. Then $\operatorname{Pr}\left(\mathcal{A}_{n}\right)$ is a good set.

ProOF: Fix $n \in \mathbb{N}$, a group $G$ and a normal abelian subgroup $H$ such that $(G: H)=n$. Let

$$
G=\bigsqcup_{i=1}^{n} H x_{i}, \quad\left(x_{1}=1\right)
$$

be a decomposition of $G$ into cosets of $H$. For each ordered pair $(i, j)$ of indices from the set $\{1, \ldots, n\}$, let

$$
S_{i j}:=\left\{\left(h_{1}, h_{2}\right) \in H \times H:\left[h_{1} x_{i}, h_{2} x_{j}\right]=1\right\} .
$$

Thus

$$
\operatorname{Pr}(G)=\frac{1}{|G|^{2}} \cdot \sum_{i, j=1}^{n}\left|S_{i j}\right|
$$


Since $H$ is abelian, it follows easily from Lemma 2.2 that, for any $g \in G$ the map $h \mapsto[h, g]$ is an endomorphism of $H$, whose kernel is $C_{H}(g)$. Let $H_{g}:=[H, g]$ and $n_{g}:=\left(H: C_{H}(g)\right)$. Thus $H_{g}$ is a subgroup of $H$ of order $n_{g}$. For each $i=1, \ldots, n$ above, let $H_{i}:=H_{x_{i}}$ and $n_{i}:=n_{x_{i}}$. For each ordered pair $(i, j)$, set $H_{i j}:=H_{i} \cap H_{j}$ and $n_{i j}:=\left|H_{i j}\right|$. Thus $n_{i j}$ is a common divisor of $n_{i}$ and $n_{j}$. Now fix a pair $(i, j)$ and set $h_{i j}:=\left[x_{j}, x_{i}\right]$. This is a fixed element of $H$. If $h_{1}, h_{2} \in H$ then, using Lemma 2.2, it is easy to check that

$$
\left[h_{1} x_{i}, h_{2} x_{j}\right]=1 \Leftrightarrow\left[h_{1}^{x}, y\right]=h_{i j}\left[h_{2}^{y}, x\right] .
$$

Let

$$
\hat{H}_{i j}:=H_{j} \cap\left(h_{i j} H_{i}\right)=\left\{h \in H_{j}: h=h_{i j} u, \text { for some } u \in H_{i}\right\} .
$$

It's easy to see that either $\hat{H}_{i j}=\phi$ or is a single coset in $H$ of the subgroup $H_{i j}$. In the former case, the right-hand side of (3.4) has no solutions. In the latter case, we can count the number of solutions as follows: first, we pick $h_{1}$ such that $\left[h_{1}^{x}, y\right] \in \hat{H}_{i j}$. The number of possible choices is just $|H| \cdot \frac{\left|H_{i j}\right|}{\left|H_{j}\right|}=|H| \cdot \frac{n_{i j}}{n_{j}}$. Having chosen $h_{1}$, we pick $h_{2}$ so that the right-hand equation in (3.4) is satisfied. The number of choices for $h_{2}$ is just $\left(H: H_{i}\right)=|H| \cdot \frac{1}{n_{i}}$. Summarising, we have shown that

$$
\left|S_{i j}\right|= \begin{cases}0, & \text { if } \hat{H}_{i j}=\phi, \\ |H|^{2} \cdot \frac{n_{i j}}{n_{i} n_{j}}, & \text { otherwise. }\end{cases}
$$

Hence, the expression (3.3) for $\operatorname{Pr}(G)$ has the form

$$
\operatorname{Pr}(G)=\frac{1}{n^{2}} \cdot \sum_{k=1}^{L} \frac{1}{x_{k}},
$$

where each $x_{k}$ is a positive integer, $x_{1}=1$ and $1 \leq L \leq n^{2}$. Here, $x_{1}$ corresponds to the term $S_{11}$ and the fact that $L$ may be less than $n^{2}$ corresponds to the fact that some of the $S_{i j}$ may be empty. Further, note that the numbers $x_{k}$ are not independent of one another, since the same can be said of the numbers $\left|S_{i j}\right|$. However, this just makes our life easier. We conclude that the set of possible values for $\operatorname{Pr}(G)$, in the notation of Lemma 2.4, is contained in the set

$$
\frac{1}{n^{2}} \cdot\left(\bigcup_{k=1}^{n^{2}} \mathcal{S}_{k}\right)
$$

Lemma 2.4 thus directly implies the claim of Lemma 3.1.

Corollary 3.2. For each $n \in \mathbb{N}$, let $\mathcal{A}^{n}$ denote the collection of all finite groups possessing an abelian subgroup of index at most $n$. Then $\operatorname{Pr}\left(\mathcal{A}^{n}\right)$ is a good set.

Proof: If $A$ is an abelian subgroup of $G$ of index at most $n$, then $\operatorname{Core}_{G}(A)$ is an abelian, normal subgroup of index at most $n$ !. Hence $\mathcal{A}^{n} \subseteq \cup_{k=1}^{n !} \mathcal{A}_{k}$ and we can apply Lemma 3.1.

We are now ready to prove the Main Theorem in a sequence of steps. Let $G$ be a non-abelian group satisfying $\operatorname{Pr}(G)>2 / 9$. By Lemma 2.1(x), we may assume that 
$Z(G) \subseteq G^{\prime}$

Step 1: Let $d$ be the minimum degree of a non-linear irreducible representation of $G$. From Lemma 2.1(vi) we deduce that either $\left|G^{\prime}\right|<8$ or $d=2$.

Step 2: First suppose $\left|G^{\prime}\right|<8$. Since $Z(G) \subseteq G^{\prime}$, it is easy to check that either $G$ is covered by parts (iii), (iv) and (v) of Lemma 2.1, or $G$ is nilpotent and a direct product of $p$-groups satisfying (2.5). It's then just a matter of verifying that the Main Theorem is satisfied in these cases.

Step 3: So we may suppose $d=2$. Let $\phi$ be an irreducible representation of $G$ of degree 2 . Let $\pi: \operatorname{GL}(2, \mathbb{C}) \rightarrow \operatorname{PGL}(2, \mathbb{C})$ be the natural projection and set $K:=\operatorname{ker}(\phi)$, $L:=\operatorname{ker}(\pi \circ \phi)$. Then $G / L$ is isomorphic to a finite subgroup of $\operatorname{PGL}(2, \mathbb{C})$, hence to one of the non-cyclic groups listed in Lemma 2.2.

First suppose that $G / L \cong A_{4}, S_{4}$ or $A_{5}$. Since $\operatorname{Pr}(G)>2 / 9$, the second and third options are immediately ruled out by (2.10) and part (viii) of Lemma 2.1. In the case of $A_{4}$, the same analysis, together with Lemma 2.1(i), implies that $K$ must be abelian. But then we can apply Lemma 3.1, and the Main Theorem is satisfied.

So we may suppose that

$$
G / K \cong Z \cdot 2 D_{n} \text {, for some } n \geq 2 \text {, where } Z \text { is a finite cyclic group. }
$$

First suppose $n \geq 3$. Then $G / L \cong D_{n}$, say

$$
G / L=<L a, L b \mid a^{n}, b^{2},(a b)^{2} \in L>.
$$

We now consider two separate cases:

CASE $1: n \geq 15$.

Since $\frac{5}{8}\left(\frac{n+6}{4 n}\right)<\frac{2}{9}$ for all $n \geq 15$, we can argue as before that $L$ must be abelian. By Lemma 2.1(ix),

$$
\frac{c}{2 n} \frac{k_{G}(L)}{|L|}>\frac{2}{9}
$$

where the number $c$ is such that every subgroup of $D_{n}$ contains at most $c$ conjugacy classes. Clearly we can take $c=n$, whence (3.11) becomes

$$
\frac{k_{G}(L)}{|L|}>\frac{4}{9}
$$

In other words, the average size of a $G$-orbit in $L$ is less than $9 / 4$. It follows easily that $a^{k} \in C_{G}(L)$ for some $k=O(1)$, independent of $n$. Let $N:=<L, a^{k}>$. Then $N$ is an abelian, normal subgroup of $G$ of bounded index, so the Main Theorem holds, by Lemma 3.1.

CASE $2: 3 \leq n \leq 14$

Then $|G / L|$ is bounded. If $(L: Z(L))$ were also bounded, then $Z(L)$ would be a normal, abelian subgroup of $G$ of bounded index and we could apply Lemma 3.1 again. 
So we may suppose $L$ is non-abelian. Since $\operatorname{Pr}(G / L) \leq \frac{5}{8}$ and since $\frac{11}{32} \times \frac{5}{8}<\frac{2}{9}$, Lemma 2.1(i) would still imply that $(L: Z(L))$ were bounded, unless $\left|L^{\prime}\right|=2$ and $L / Z(L) \cong C_{2}^{2 s}$ for some $s \in \mathbb{N}$. Thus, $L^{\prime} \subseteq Z(G)$. We can still apply Lemma 2.1(ix) to conclude that

$$
\frac{k_{G}(L)}{|L|}>\frac{8}{5} \times \frac{2}{9}=\frac{16}{45}
$$

In other words, the average size of a $G$-orbit in $L$ is less than $45 / 16$. This must imply that $\left(L: L_{1}\right)$ is bounded, where $L_{1}=\left\{x \in L:[G, x] \subseteq L^{\prime}\right\}$. Now $G^{\prime} / L^{\prime} \cong\left(G / L_{1}\right)^{\prime}$. By Lemma 2.1(i), either $\left|G^{\prime}\right| \leq 6$ or $\operatorname{Pr}\left(G / L_{1}\right) \leq \frac{7}{16}$. The first alternative takes us back to Step 2. From the second alternative and Lemma 2.1(viii) we conclude that $\operatorname{Pr}\left(L_{1}\right)>\frac{32}{63}=\frac{1}{2}+\frac{1}{126}$ and hence, by Lemma 2.1(i), that $\left(L_{1}: Z\left(L_{1}\right)\right)$ is bounded. But since $(G: L)$ and $\left(L: L_{1}\right)$ are also bounded, we conclude that $\left(G: Z\left(L_{1}\right)\right)$ is bounded and we can apply Lemma 3.1 one more time to conclude the analysis of CASE 2.

We are now left with the possibility that $n=2$ in (3.9). Set $G / K:=Q$. Then $\left|Q^{\prime}\right|=2$, $Q / Z(Q) \cong C_{2} \times C_{2}$ and $Z(Q)$ is a cyclic group. A priori, the order of $Q$ may be unbounded, but the crucial thing is that $Q$ has a cyclic subgroup of bounded index. Let $q \in G$ be such that $K q$ generates $Z(Q)$. Since $\operatorname{Pr}(Q)=5 / 8$ we can repeat the analysis from CASE 2 above to conclude that either $\left|G^{\prime}\right| \leq 6$ or $K$ contains an abelian subgroup $K_{2}$ of bounded index such that $K_{2} \triangleleft G$. So we may suppose the latter holds. Lemma 2.1(ix) still applies and, as in (3.13), we have that the average size of a $G$-orbit in $K_{2}$ is less than $45 / 16$. Let $K_{3}:=\left\{x \in K_{2}:\left(G: C_{G}(x)\right) \leq 2\right\}$. A priori, $K_{3}$ may not be a subgroup of $K_{2}$, however we must have that $\left|K_{3}\right| /\left|K_{2}\right|$ is bounded away from zero. In addition, since $\left(G: C_{G}(x)\right) \leq 2$ for all $x \in K_{3}$, it follows that $K_{3} \subseteq C_{g}\left(q^{2}\right)$. Let $K_{4}:=C_{G}\left(q^{2}\right) \cap K_{2}$ and $A:=<K_{4}, q^{2}>$. Then $A$ is an abelian subgroup of $G$ of bounded index, and hence we can apply Corollary 3.2. This completes the proof of the Main Theorem.

\section{Discussion}

It is not true that if $\operatorname{Pr}(G)$ is bounded away from zero, then $G$ contains a (normal) abelian subgroup of bounded index. Indeed, by Lemma 2.1(i), we see that this already fails for groups satisfying $\operatorname{Pr}(G)>1 / 2$. So we cannot prove Joseph's first two conjectures simply by using Lemma 3.1. Indeed, Theorem 1.1 seems to give the strongest possible structural result about groups for which $\operatorname{Pr}(G)$ is bounded away from zero. Note that the structure described there includes the case when $\left|G^{\prime}\right|$ is bounded. Indeed, the strategy of our proof in the previous section began by appealing to Lemma 2.1(vi), which says that if $\operatorname{Pr}(G)$ is bounded away from zero, then either $\left|G^{\prime}\right|$ or the minimum degree of a non-linear irreducible representation of $G$ is bounded. In the latter case, it is also interesting that a classical result of Jordan (see $[\mathbf{I}]$, Theorem 14.12) says that there is a function $f: \mathbb{N} \rightarrow \mathbb{N}$ such that a finite subgroup of $\operatorname{GL}(n, \mathbb{C})$ must contain an abelian, normal subgroup of index at most $f(n)$. However, it is unlikely that we can say anything more than Theorem 1.1 about the structure of $G$ in general]. Therefore, it

\footnotetext{
${ }^{7}$ Neumann's proof does not use any representation theory, though he does make use of the wellknown fact that if the sizes of the conjugacy classes in a group are bounded, then so is the size of the full commutator subgroup.
} 
seems a crucial step in the analysis of Joseph's conjectures is to see if the sets $\operatorname{Pr}\left(\mathcal{C}_{n}\right)$ are good, where $\mathcal{C}_{n}$ is the collection of all finite groups $G$ for which $\left|G^{\prime}\right| \leq n$. It is still not obvious to us how one would get from there and Lemma 3.1 to a full proof of Joseph's first two conjectures, but at least we have provided a possible roadmap.

Finally, we have not said anything in this paper about Joseph's third conjecture, which seems more mysterious to us.

\section{ACKNOWLEDGEMENT}

I thank Des MacHale for helpful discussions and for drawing my attention to several of the papers in the bibliography below.

\section{REFERENCES}

[C] A. Castelaz, Commutativity degree of finite groups, M.A. thesis, Wake Forest University (2010).

[DN] A.K. Das and R.K. Nath, A characterisation of certain finite groups of odd order, Math. Proc. R. Ir. Acad. 111A (2011), No.2, 69-78.

[ET] P. Erdős and P. Turán, On some properties of a statistical group theory, IV, Acta Math. Acad. Sci. Hung. 19 (1968), 413-435.

[G] W.H. Gustafson, What is the probability that two group elements commute?, Amer. Math. Monthly 80 (1973), 1031-1034.

[GMMPS] N. Gavioli, A. Mann, V. Monti, A. Previtali and C.M. Scoppola, Groups of prime order with many conjugacy classes, J. Algebra 202 (1998), No.1, 129-141.

[GR] R.M. Guralnick and G.R. Robinson, On the commuting probability in finite groups, J. Algebra 300 (2006), No.1, 509-528.

[I] M. Isaacs, Character theory of finite groups, Academic Press, New York (1976).

[J1] K.S. Joseph, Commutativity in non-abelian groups, Ph.D. thesis, UCLA (1969).

[J2] K.S. Joseph, Several conjectures on commutativity in algebraic structures, Amer. Math. Monthly 84 (1977), 550-551.

[Ke] T.M. Keller, Lower bounds for the number of conjugacy classes of finite groups, Math. Proc. Cambridge Philos. Soc. 147 (2009), No.3, 567-577.

[K1] F. Klein, The icosahedron and the solution of equations of the fifth degree, Dover, New York (1956).

[L] P. Lescot, Isoclinism classes and commutativity degrees of finite groups, J. Algebra 177 (1995), No.3, 847-869.

[Neu] P.M. Neumann, Two combinatorial problems in group theory, Bull. London Math. Soc. 21 (1989), No.5, 456-458.

[New] M. Newman, A bound for the number of conjugacy classes in a group, J. London Math. Soc. 43 (1968), 108-110.

[NiS] Á. Ní Shé, Commutativity and generalisations in finite groups, Ph.D. thesis, National University of Ireland, Cork (2000).

[PS] V. Ponomarenko and N. Selinski, Two semigroup elements can commute with any positive rational probability. Preprint available from author homepage: http://www-rohan.sdsu.edu/ vadim/research.html

[R] D.J. Rusin, What is the probability that two elements of a finite group commute?, Pacific J. Math. 82 (1979), No.1, 237-247.

[S] I. Schur, Über die Darstellung der endlichen Gruppen durch gebrochene lineare Substitutionen, J. Reine Angew. Math. 127 (1904), 20-50. 
LIMIT POINTS IN THE RANGE OF THE COMMUTING PROBABILITY FUNCTION ON FINITE GROURS

Department of Mathematical Sciences, Chalmers University Of Technology and University of GothenbURG, 41296 Gothenburg, SWEDEN

E-mail address: hegarty@chalmers.se 